side of the observed Lyman $\alpha$ line, and hundreds of angströms away. An accurate study of the continuum in such cases would be very important.

Finally, the chief argument against the predominantly cosmological nature of the red-shift $z$ is that the plot of $z$ against $m_{v}$ looks so much like a mere scatter-diagram ${ }^{3}$. Any expected relation between these quantities can be confused by several familiar effects (intrinsic scatter, spectral energy-distribution, instrumental cut-offs, and so on). If the hypothesis of the jet is valid, it complicates the situation further by making $m_{v}$ depend on the aspect of the source, and to some extent by the possibility that the observed red-shift is not precisely the cosmological red-shift. In fact, there are so many confusing factors that the outcome is bound to look like a scatter diagram unless the sources can be classified in considerable detail.

The idea here put forward is, basically, only a slight modification of the current picture, but we see that it might cause a surprising variety of observed features to fall into place; also a number of observational tests can be proposed.

Astronomy Centre,

W. H. MCCREA

University of Sussex.

Received March 20, 1968.

${ }^{1}$ Burbidge, G. R., and Burbidge, M., Quasi-8tellar Objects (Freeman, San Francisco and London, 1967)

${ }^{2}$ McCrea, W. H., Nature, 213, 239 (1967); McCrea, W. H., Science, 157, 400 (1967).

${ }^{3}$ Burbidge, G. R., and Hoyle, F., Nature, 216, 351 (1967).

\section{Far Ultraviolet Radiation from the Milky Way}

THe far ultraviolet photometer mounted on Venera-4, launched on June 12, 1967, scanned the sky to measure the ultraviolet radiation from the Milky Way, which was discovered by Kurt1. The observations were made in two spectral intervals, the first including the hydrogen Lyman $\alpha$ line $(\lambda 1050-1340 \AA)$ and the second off the Lyman $\alpha \operatorname{lin} \theta(\lambda 1225-1340 \AA)$.

The diameters of the photometer fields of view were $10^{\circ}$ and $20^{\circ}$, respectively. The scanning was made along a small circle about $140^{\circ}$ diameter with the centre at the antisolar point, looking from the space craft, with coordinates $\alpha=21^{\mathrm{h}} 44^{\mathrm{m}}, \delta=-16^{\circ} 15^{\prime}$. The scanning circle in the new galactic co-ordinates $(l \mathrm{II}, b \mathrm{II})$ is shown in Fig. 1. The photometer field of view crossed the Milky Way belt twice; near the constellations Cassiopeia and Cygnus in the northern hemisphere and near the constellation Lupus in the southern hemisphere. The four scans

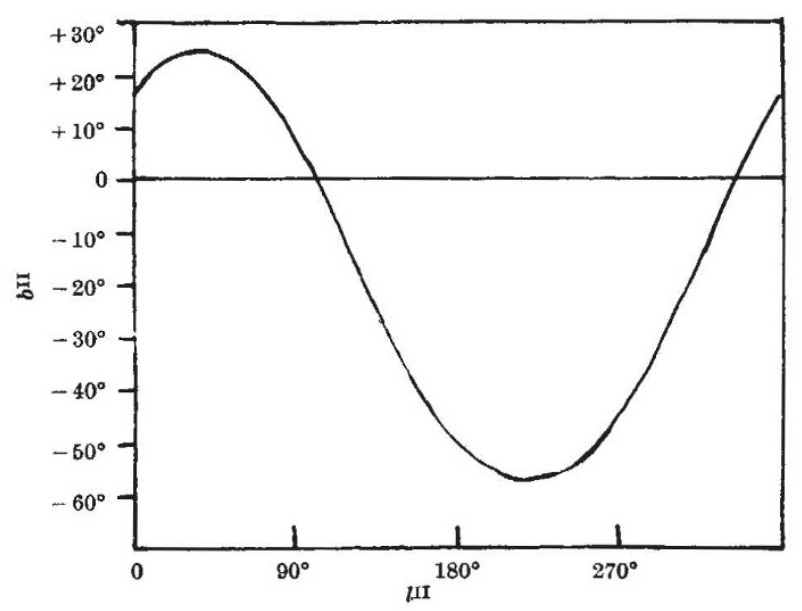

Fig. 1. Scanning circle in the now galactic co-ordinates $\left(l^{\mathrm{m}}, b^{\mathrm{nt}}\right)$.

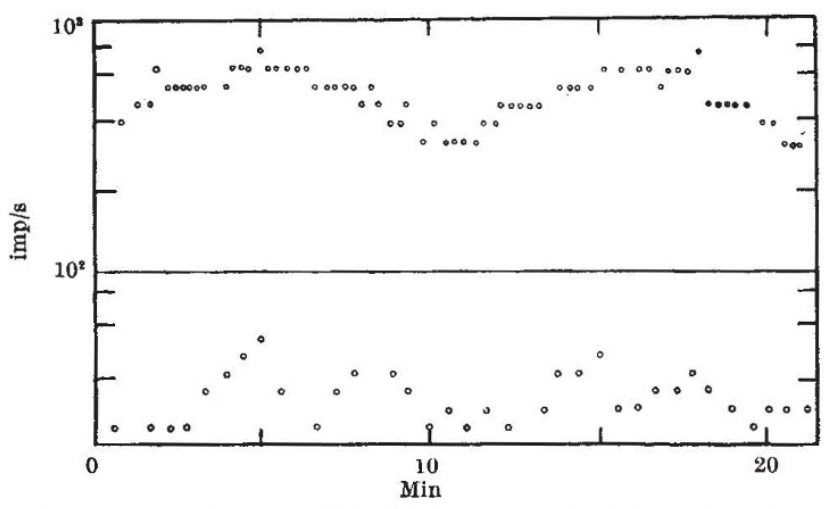

Fig. 2. One of the scans, obtained on August 16,1967. The circles are the dimensions of the fleld of view.

obtained are quite identical in the first spectral interval, containing the Lyman $\alpha$ line, and are similar in the second. Fig. 2 shows two scans in both spectral intervals, the period being $10 \mathrm{~min}$. The maximum signal in the first spectral interval is about 25 times greater than that in the second interval outside the Lyman $\alpha$ line. About half the signal in spectral interval $\lambda 1225-1340 \AA$ is caused by the cosmic ray background. Taking into account the permeability of the additional $\mathrm{CaF}_{2}$ filter, which cuts off radiation with $\lambda<1225 \AA$, the photon counter officiency and the difference in the fields of view, the ratio of the fluxes is

$$
F(\lambda 1050-1340 \AA) / F(\lambda 1225-1340 \AA) \simeq 50
$$

The high value of the ratio shows that the radiation is observed in a narrow band and that its intensity is about $200 \mathrm{r}$.

The nature of the emission was considered by Kurt and Sunajev, who discuss the difficulties of interpreting it as the transformed radiation of early spectral type stars. At the same time the radiation in the $(\lambda 1225$ $1340 \AA$ ) band is undoubtedly the integrated radiation of hot stars. It is noted in refs. 1 and 2 that the flux ratio (by unit interval of wavelength) does not exceed $10^{-2}$ and is much smaller than the calculated values of Zimmermann $^{3}$. The new observations give the value $4 \times 10^{-2}$.

The most conspicuous details in Fig. 2 are the strong emission near $b \mathrm{II}=0^{\circ}, l \mathrm{II}=100^{\circ}$ and almost complete absence of radiation near $b \mathrm{II}=0^{\circ}, l \mathrm{II}=300^{\circ}$ (the southern hemisphere). Stecher ${ }^{4}$ shows that light absorption by dust in the investigated spectral interval does not permit observations at distances greater than 1-3 kpc. According to $\mathrm{Bok}^{5}$, the Perseus arm of the galaxy and even the nearest arm (Carina-Cygnus) are located at this distance. There is a void in the direction $l \mathrm{II}=300^{\circ}$ up to a distance of about $5 \mathrm{kpc}$. Kurt and Sunajev showed, however, that only quanta shifted from the Lyman $\alpha$ line by not less than $\sim 5 \AA$ can propagate at such a distance, which involves velocities exceeding $1,000 \mathrm{~km} \mathrm{~s}^{-1}$. In the case of multiple dispersion, the quanta are absorbed by dust and diffuse small distances. Because of this, the hypothesis that the observed Lyman $\alpha$ emission originates in the peripheral parts of the solar system, where the solar wind is controlled by the galactic magnetic field ${ }^{2}$, is quite attractive.

\section{G. KURT}

S. B. Dostovalov

Sternberg State Astronomical Institute, Moscow, USSR.

Received March 19, 1968.

${ }^{1}$ Kurt, V. G., Astron. Circ. Acad. Sci. USSR, 439, 2 (1967).

2 Kurt, V. G., and Sunajev, R. A., Astr. Zh., 44, 1157 (1967).

- Zimmermann, H., Astro. Nachr., 288, 95 (1964).

4 Stecher, T. R., Ap. J., 142, 1683 (1965).

- Bok, B. I., Observatory, 79, 58 (195):. 